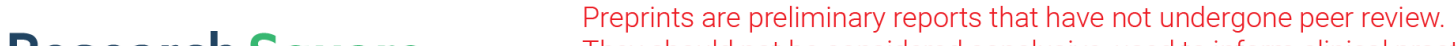 They should not be considered conclusive, used to inform clinical practice, or referenced by the media as validated information. \\ Colossal and Reversible Barocaloric Effect in Phase Change Materials n-Alkanes
}

\section{Jianchao Lin ( $\boldsymbol{\nabla}$ jclin@issp.ac.cn )}

Institute of Solid State Physics, Chinese Academy of Sciences

\section{Peng Tong}

Institute of Solid State Physics

\section{Kun Tao}

Institute of Solid State Physics, Chinese Academy of Sciences

\section{Wenjian Lu}

Key Laboratory of Materials Physics, Institute of Solid State Physics, Chinese Academy of Sciences, Hefei 230031, People's Republic of China

\section{Xuekai Zhang}

Institute of Solid State Physics, Chinese Academy of Sciences

\section{Wenhai Song}

Institute of Solid State Physics, Chinese Academy of Sciences

\section{Yuping Sun}

Institute of Solid State Physics Chinese Academy of Sciences Hefei https://orcid.org/0000-0003-2477$285 X$

\section{Article}

Keywords: emergent cooling technologies, caloric effect, colossal barocaloric effect

Posted Date: May 6th, 2021

DOI: https://doi.org/10.21203/rs.3.rs-257277/v1

License: (1) This work is licensed under a Creative Commons Attribution 4.0 International License.

Read Full License

Version of Record: A version of this preprint was published at Nature Communications on February 1st, 2022. See the published version at https://doi.org/10.1038/s41467-022-28229-4. 


\title{
Colossal and Reversible Barocaloric Effect in Phase Change Materials $n$-Alkanes
}

\begin{abstract}
The emergent cooling technologies based on the caloric effect provide a green alternative to the conventional vapor-compression one which brings about the serious environment problem. However, the existing caloric materials are much inferior to their traditional counterparts in cooling performance. Here we report the colossal barocaloric effect in liquid-solid-transition materials, i.e. $n$-alkanes. Their excellent cooling performance is superior to those for existing caloric materials and comparable to those of traditional refrigerants. Theoretical calculations suggest the liquid state $n$ alkanes has huge configuration entropy characterized by large dispersion of bond lengths. Appling pressure significantly reduces the configuration entropy and eventually induces the liquid-solid-transition, leading to the colossal barocaloric effect. This work provides promising refrigerants for caloric cooling technology, and opens a new avenue for exploring colossal barocaloric materials.
\end{abstract}

Refrigeration and cooling are pervasive in modern society, covering the fields including food storage, air condition, industrial manufacture, medical treatment, etc ${ }^{1,2}$. The vast majority of the cooling equipment depends on conventional refrigeration technologies, which are based on compression cycles of powerful greenhouse gases, e.g., hydrofluorocarbons (HFCs) and hydrochlorofluorocarbons (HCFCs) ${ }^{1-4}$. Those greenhouse gases have great global warming potentials thousands of times greater than $\mathrm{CO}_{2}$ and long atmospheric lifetime for several decades ${ }^{1,3}$. According to the Kigali Amendment, the phase-down of the greenhouse gases is mandated ${ }^{1,3,4}$. Paradoxically, the demand for cooling will continue to increase in the seeable future because of population increase, urbanization, improvement in the quality of life, etc. Although have been continuously optimized, the environmentally harmful refrigerants seem unavoidable in present vapor-compression technologies ${ }^{2,4}$. Therefore, it is urgent to develop alternative cooling technologies, where the related refrigerants should be clean and environmentally friendly.

Solid-state caloric (SSC) effects provide an emergent refrigeration technology, which refer to an isothermal entropy change and adiabatic temperature change in response to a variety of external fields, often being associated with first-order phase transition in solid materials ${ }^{5-7}$. Depending on the types of external fields, such as magnetic field, electrical field, uniaxial stress and hydrostatic pressure, the SSC effects are sorted into magnetocaloric, electrocaloric, elastocaloric and barocaloric (BC) effects, respectively ${ }^{5-7}$. Unlike the commercial refrigerants currently used in vaporcompression cycles, the SSC materials have little environmental impact. However, for most SSC materials, the isothermal entropy change is on the order of $10-100 \mathrm{~J} \mathrm{~kg}^{-1} \mathrm{~K}^{-}$

${ }^{1}$, which is far below those of commercial refrigerants, e.g., $\sim 520 \mathrm{~J} \mathrm{~kg}^{-1} \mathrm{~K}^{-1}$ for $\mathrm{R} 134 \mathrm{a}$ (one of the most commonly used HFCs) ${ }^{8}$. The pretty low entropy change indicates a significantly smaller refrigeration capacity, which will inevitably restrict their potential applications. Until very recently, breakthrough achieved in plastic crystals significantly reduced the gap of cooling capacity between the SSC refrigerants and the traditional ones $^{8-11}$. 
Matters have various states (e.g., gas, liquid and solid) depending on the strength of the interaction among molecules. The stronger the intermolecular interaction, the more ordered the matter state, the lower the entropy. So, the colossal entropy change can be anticipated when a matter transforms from one state to another. It is based on the gasliquid transformation that the traditional vapor-compression technology realizes the strong cooling capacity. The reported SSC effect is mainly associated with the transformation between two "solid" states with notably different degrees of lattice, spin, polar or electron ordering ${ }^{5-21}$. Therefore, it is a big challenge for SSC materials to acquire as good refrigeration performance as conventional refrigerants. Alike the gasliquid transitions, the liquid-solid (LS) transitions also exhibit huge entropy change ${ }^{22-}$ 26. Moreover, the LS transition is often characterized by a large volume change since the density changes, which is thus sensitive to external pressure and could be utilized for realizing colossal $\mathrm{BC}$ effects ${ }^{26-28}$. As typical LS-transition materials, the $n$-alkanes with general chemical formula $\mathrm{C}_{n} \mathrm{H}_{2 n+2}$ have been widely used in food, medicine, packaging, fruit preservation and other fields, indicating good environmental friendliness. Here, we evaluated the colossal BC effect associated with the LS transition in $\mathrm{C}_{n} \mathrm{H}_{2 n+2}(n=14,16,18)$. Under an external pressure of $100 \mathrm{MPa}$, a high adiabatic temperature change $\left(\Delta T_{\mathrm{d}}\right) \sim 18 \mathrm{~K}$ was achieved by direct method, which is doubled when the applying pressure is increased to $200 \mathrm{MPa}$. Moreover, the reversible entropy change $\left(\Delta S_{\mathrm{r}}\right)$ reaches $\sim 700 \mathrm{~J} \mathrm{~kg}^{-1} \mathrm{~K}^{-1}$ under only $\sim 50 \mathrm{MPa}$. Both the $\Delta T_{\mathrm{d}}$ and $\Delta S_{\mathrm{r}}$ of $n$ alkanes are well exceeding those of existing SSC materials, promising for caloric cooling applications. The colossal BC effect can be understood in terms of pressureinduced drastic change of configuration entropy in the liquid state.

The temperature variation of $n$-alkanes as function of time, $T(\mathrm{t}) \mathrm{s}$, were measured by direct method under different pressures (Supplementary Fig. 1-3). The results of $\mathrm{C}_{18} \mathrm{H}_{38}$ at selected temperatures were shown in Fig. 1A-C. In the solid state (e.g., at $294 \mathrm{~K}$ ), the sample's temperature rises when pressure is applied and decreases when pressure is released, corresponding to the exothermic and endothermic peaks in the $T(\mathrm{t})$ curves, respectively. Both the endothermic and exothermic peaks are gradually enhanced with increasing the pressure up to $500 \mathrm{MPa}$. When the measurement temperature is set at $305 \mathrm{~K}$ which is slightly higher than the LS transition temperature ( $\left.T_{\mathrm{LS}} \sim 301.3 \mathrm{~K}\right)$, under pressures at $\geq 100 \mathrm{MPa}$, the exothermic peak during the pressure-loading process is sharp and gets further enhanced as the pressure increase ${ }^{25}$. In contrast, the endothermic peak during the pressure unloading process always presents shape distortion and broadening. When the temperature is set well above $T_{\mathrm{LS}}$ (e.g., at $356 \mathrm{~K}$ ), the endothermic peak shows an abrupt enhancement at pressure $367 \mathrm{MPa}$. However, the exothermic peak is distorted into a small peak with a long tail. The above behaviors were also observed for $\mathrm{C}_{16} \mathrm{H}_{34}$ and $\mathrm{C}_{14} \mathrm{H}_{30}$ samples, as shown in Supplementary Fig. 2 and 3 , respectively.

The hydrostatic pressure can significantly enhance $T_{\mathrm{LS}}$ of $\mathrm{C}_{n} \mathrm{H}_{2 n+2}{ }^{26-27}$. Therefore, when $\mathrm{C}_{n} \mathrm{H}_{2 n+2}$ lies in liquid state, the strong pressure could drive the mutual transformation between liquid and solid state. So, the extra enhancement of the exothermic and endothermic peaks (Fig. 1A-C) can be attributed to the transition from liquid to solid state under pressure loading and the reverse transition as a result of 
pressure release, respectively. The magnitude of the pressure depends directly on the temperature. The higher the temperature is, the greater the pressure is required. So, at $305 \mathrm{~K}$ only $100 \mathrm{MPa}$ can trigger the phase transition, but at $356 \mathrm{~K}$ a high pressure of $367 \mathrm{MPa}$ is required. In the solid state, like at $294 \mathrm{~K}$, the pressure can't drive any phase transformations, and thus the abnormal increase of sample's temperature is absent. The different behaviors of endothermic and exothermic peaks, sharp and single or broad and distorted, can be understood in terms of the cooperation and competition between pressure and temperature (Supplementary Note 1).

Since the present testing apparatus cannot be fully insulated, the precision of adiabatic temperature change $\left(\left|\Delta T_{\mathrm{d}}\right|\right.$, i.e. the magnitude of temperature jump in the $T(\mathrm{t})$ curves) will be strongly affected by the exothermic and endothermic time. By analyzing the original data (Supplementary Note 1), the reliable $\left|\Delta T_{\mathrm{d}}\right|$ for $\mathrm{C}_{18} \mathrm{H}_{38}, \mathrm{C}_{16} \mathrm{H}_{34}$ and $\mathrm{C}_{14} \mathrm{H}_{30}$, are shown in Fig. 1D, Fig. 1E and Supplementary Fig. 3D, respectively. For all samples, $\left|\Delta T_{\mathrm{d}}\right|$ increases almost linearly with pressure when the pressure is too small to drive the LS phase transition. When the pressure is larger than the critical value, the extra contribution from the LS phase transition comes in and results in an abrupt increase of $\left|\Delta T_{\mathrm{d}}\right|$. In this case, a large $\left|\Delta T_{\mathrm{d}}\right|$ can be accepted under the low pressure as soon as setting the temperature slightly above $T_{\text {LS. }}$. When the pressure is increased to a high value, a much larger $\left|\Delta T_{\mathrm{d}}\right|$ was obtained. For example, $\left|\Delta T_{\mathrm{d}}\right|$ reaches $\sim 45 \mathrm{~K}$ under $300 \mathrm{MP}$ and $\sim 57 \mathrm{~K}$ under $400 \mathrm{MPa}$ for present samples. But in the solid state, a high pressure of $500 \mathrm{MPa}$ can only induce a small $\left|\Delta T_{\mathrm{d}}\right|(\sim 10 \mathrm{~K})$.

Based on the DSC results at ambient pressure (Supplementary Fig. 4) and DTA signals recorded at different pressures (Supplementary Fig. 5), the temperature dependent entropy change solely due to the LS phase transition (denoted as $\Delta S_{\mathrm{LS}}$ ) for $\mathrm{C}_{18} \mathrm{H}_{18}$ and $\mathrm{C}_{16} \mathrm{H}_{34}$ can be obtained (Supplementary Fig. 6). In combination with the specific heat results reported ${ }^{25}$, the temperature dependent total entropy, $\Delta S_{\mathrm{t}}(\mathrm{T})$, was constructed at different pressures (Supplementary Fig. 7). Finally, the pressure driven entropy changes, $\Delta S_{\mathrm{ir}}(\mathrm{T})$ (the irreversible one), were derived and plotted in Supplementary Fig. 8. The derived reversible entropy changes, $\Delta S_{\mathrm{r}}(\mathrm{T}) \mathrm{s}$, were shown in Fig. $1 \mathrm{~F}$ and Fig. $1 \mathrm{G}$ for $\mathrm{C}_{18} \mathrm{H}_{18}$ and $\mathrm{C}_{16} \mathrm{H}_{34}$, respectively. A small pressure of about 40 $\mathrm{MPa}$ and $58 \mathrm{MPa}$ can nearly activate the total $\Delta S_{\mathrm{r}}(\mathrm{T})$ of $698 \mathrm{~J} \mathrm{~K}^{-1} \mathrm{~kg}^{-1}$ and $759 \mathrm{~J} \mathrm{~kg}^{-1} \mathrm{~K}^{-}$ ${ }^{1}$ for $\mathrm{C}_{18} \mathrm{H}_{38}$ and $\mathrm{C}_{16} \mathrm{H}_{36}$, respectively. With further increasing pressure, $\Delta S_{\mathrm{r}}(\mathrm{T})$ is increased slightly to $779 \mathrm{~J} \mathrm{~kg}^{-1} \mathrm{~K}^{-1}$ at $232 \mathrm{MPa}$ for $\mathrm{C}_{18} \mathrm{H}_{38}$, and $808 \mathrm{~J} \mathrm{~kg}^{-1} \mathrm{~K}^{-1}$ at 152 $\mathrm{MPa}$ for $\mathrm{C}_{16} \mathrm{H}_{34}$. Furthermore, the temperature window of the colossal and reversible $\Delta S_{\mathrm{r}}(\mathrm{T})$, i.e., the "platform" in the $\Delta S_{\mathrm{r}}(\mathrm{T})$ curve, expands with increasing pressure. For $\mathrm{C}_{18} \mathrm{H}_{38}$, it reaches $\sim 38 \mathrm{~K}$ at $232 \mathrm{MPa}$, while for $\mathrm{C}_{16} \mathrm{H}_{34}$ it reaches $\sim 22 \mathrm{~K}$ at $152 \mathrm{MPa}$. Such a temperature window for reversible BC effect is much wider than that reported in other colossal BC materials, such as NPA (less than $10 \mathrm{~K}$ at $330 \mathrm{MPa}$ ) ${ }^{10}$. The wide temperature window is a premise for designing cooling devices with a broad operating temperature range. Additionally, we note that $\Delta S_{\mathrm{r}}(\mathrm{T})$ in Supplementary Fig. 9 is underestimated because the calculated $S_{\mathrm{t}}(\mathrm{T})$ curves are based on the specific heat at ambient condition (Supplementary Note 2) since the pressure-dependent specific heat is not available. 
The maximum $\left|\Delta T_{\mathrm{d}}\right|$ and $\left|\Delta S_{\mathrm{r}}\right|$ values as a function of pressure were summarized and shown in Fig. 2A and Fig. 2B for $\mathrm{C}_{n} \mathrm{H}_{2 n+2}(n=14,16$ and 18) along with those reported for existing $\mathrm{BC}$ materials ${ }^{10,13-17,20,21,29-39}$. Here, the isothermal entropy change in references is mainly obtained by indirect method or quasi-direct method ${ }^{6}$. According to different treatment methods, reversible entropy change $\left(\left|\Delta S_{\mathrm{r}}\right|\right)$ and non-reversible entropy change $\left(\left|\Delta S_{\mathrm{ir}}\right|\right)$ can be obtained. In literatures, the adiabatic temperature changes were measured either by direct measurement $\left(\left|\Delta T_{\mathrm{d}}\right|\right)$, or by quasi-direct method (reversible adiabatic temperature changes $\left|\Delta T_{\mathrm{r}}\right|$ and non-reversible adiabatic temperature changes $\left.\left|\Delta T_{\text {ir }}\right|\right)^{6}$. In general, $\left|\Delta S_{\text {ir }}\right|$ and $\left|\Delta T_{\text {ir }}\right|$, which ignore the inherent thermal hysteresis effect, are easily overestimated compared with $\left|\Delta S_{\mathrm{r}}\right|$ and $\left|\Delta T_{\mathrm{r}}\right|$, while $\left|\Delta T_{\mathrm{d}}\right|$ is often less than the real value as it is hard to get a fully adiabatic environment.

Even so, the maximum values of $\left|\Delta S_{\mathrm{r}}\right|$ and $\left|\Delta T_{\mathrm{d}}\right|$ (i.e. $\left|\Delta S_{\mathrm{r}}^{M}\right|$ and $\left|\Delta T_{\mathrm{d}}^{M}\right|$ ) for $\mathrm{C}_{n} \mathrm{H}_{2 n+2}$ are remarkably higher than those of the reported BC materials. Acetoxy Silicone Rubber exhibits large $\left|\Delta T_{\mathrm{r}}^{M}\right|$ comparable to $\left|\Delta T_{\mathrm{d}}^{M}\right|$ of $\mathrm{C}_{n} \mathrm{H}_{2 n+2}$ but has much smaller $\left|\Delta S_{\mathrm{r}}^{M}\right|{ }^{37}$. Some plastic crystals (e.g., NPA and PG) have high $\left|\Delta S_{\mathrm{r}}^{M}\right|$ next to that of $\mathrm{C}_{n} \mathrm{H}_{2 n+2}$ at pressures above $200 \mathrm{MPa}$, but relatively small adiabatic temperature change values ${ }^{10}$. Overall, compared with the other BC materials, $\mathrm{C}_{n} \mathrm{H}_{2 n+2}$ shows more excellent $\mathrm{BC}$ performance under the pressure window tested, especially in the low-pressure range. The $\left|\Delta S_{\mathrm{r}}\right|$ of $\mathrm{C}_{n} \mathrm{H}_{2 n+2}$ under $50 \mathrm{MPa}$ reaches $\sim 700 \mathrm{~J} \mathrm{~kg}^{-1} \mathrm{~K}^{-1}$, which already lies in the scope of entropy change for commercial Freon-based refrigerants and is even larger than that for the widely used $\mathrm{R} 134 \mathrm{a}\left(\sim 520 \mathrm{~J} \mathrm{~kg}^{-1} \mathrm{~K}^{-1}\right)^{8}$. The excellent low-pressure BC performance can ensure a strong cooling ability for the massive loading, which is beneficial to their practical applications as caloric refrigerants.

To give an insight into the mechanism of the colossal BC effect in $n$-alkanes, we performed the theoretical calculation for $\mathrm{C}_{18} \mathrm{H}_{38}$ by combining the classical molecular dynamics (MD) simulation ${ }^{40-43}$ and first-principles calculation based on density functional theory (DFT) ${ }^{44,45}$. The coexisting solid-liquid phases was used to simulate the melting temperature of $\mathrm{C}_{18} \mathrm{H}_{38}$, since the two-phase MD method is more accurate for the melting simulation ${ }^{42,43}$. To acquire the exact thermodynamic data of $\mathrm{C}_{18} \mathrm{H}_{38}$, onephase MD method was employed and the related temperature $\left(T_{M D}^{S}\right)$ is larger than the real temperature due to the superheating problem. Figure $3 \mathrm{~A}$ shows that the evolutions of $T_{\mathrm{LS}}$ and $\Delta S_{\mathrm{LS}}$ (calculated from the enthalpy change shown in Supplementary Fig. 10) with pressure present a high consistency with the experiment results ${ }^{26,27}$ This indicates the rationality of the relevant models and parameters adopted in the present MD simulation. The previous studies definitely indicate that DFT is highly reliable in estimating the lattice vibration entropy $\left(S_{\mathrm{vib}}\right)$ of crystalline materials ${ }^{46}$. Therefore, the $S_{\text {vib }}$ of $\mathrm{C}_{18} \mathrm{H}_{38}$ in solid state was estimated by this mean and compared with the total entropy $\left(S_{\mathrm{t}}\right)$ obtained by experiments. The results indicate the $S_{\text {vib }}$ always contributes dominantly to $S_{\mathrm{t}}$ for solid $\mathrm{C}_{18} \mathrm{H}_{38}$, while its proportion decreases as the temperature is raised (Fig. 3B). At $100 \mathrm{~K}, S_{\mathrm{vib}}$ accounts for about $94.6 \%$ of $S_{\mathrm{t}}$. When the temperature 
is close to $T_{\mathrm{LS}}$, i.e., $290 \mathrm{~K}$, the value drops to $92.3 \%$, indicating the involvement of other contributions than the lattice vibrations.

The $n$-alkanes have a simple chain structure in solid state (Fig. 3C). As shown in Supplementary Fig. 11, the spatial distribution of the bond lengths $(\mathrm{C}-\mathrm{C}$ and $\mathrm{C}-\mathrm{H})$ is enhanced upon heating. Accordingly, when heating towards $T_{\mathrm{LS}}$, the long-range structure is gradually collapsed (Fig. 3D) and the configurational entropy increases, which is in accordance with the DFT results. After entering the liquid state, the irregular vibrations of the $\mathrm{C}$ and $\mathrm{H}$ atoms are significantly enhanced (Supplementary Video 1). The long-range structural order is completely lost (Fig. 3E) and bond lengths are highly dispersed, corresponding to an extremely high configuration entropy. It is the remarkable structural difference between solid and liquid states that results in the different pressure-response behaviors, which will be discussed in the following paragraph.

At $T_{M D}^{S}=300 \mathrm{~K}, \mathrm{C}_{18} \mathrm{H}_{38}$ is in the solid state and the structure is well ordered under $0 \mathrm{MPa}$. Increasing the pressure to $200 \mathrm{MPa}$, both the material structure and RDF curves keep unchanged (Fig. 3C and Fig. 3F), indicating that the solid state is insensitive to external pressure. When $T_{M D}^{S}$ is increased to $400 \mathrm{~K}$, which is close to $T_{\mathrm{LS}}$, the molecular chains show a weak twisting, but each molecule is relatively independent and does not twine with the others (Fig. 3D). The RDF peaks are broadened relative to those at $T_{M D}^{S}=300 \mathrm{~K}$ (Supplementary Fig. 11). At $T_{M D}^{S}=400 \mathrm{~K}$, applying $200 \mathrm{MPa}$ makes the structure more ordered than that at $0 \mathrm{MPa}$ (Fig. 3D). Accordingly, RDF shows visible response to this pressure (Fig. 3G). When $T_{M D}^{S}$ was further increased to $500 \mathrm{~K}, \mathrm{C}_{18} \mathrm{H}_{38}$ is completely in liquid state. Due to the strong atomic irregular vibration (Supplementary Video 1), the molecular chains are severely twisted and entangled with each other (Fig. 3E), and the RDF peaks are highly collapsed and broadened (Supplementary Fig. 11). At $200 \mathrm{MPa}$, the winding of molecular chains is weakened and all RDF peaks are significantly narrowed (Fig. 3H), which should be correlated with the suppressed atomic irregular vibrations. Accordingly, the configuration entropy is greatly suppressed even though a LS phase transition is not yet triggered at this pressure. This explains the considerably large incipient BC effect in the liquid state that we observed under pressures less than the critical value (e.g., Fig. 1D and E). When the pressure reaches the critical value, the $n$-alkanes undergo the LS transition, the configurational entropy will be greatly suppressed as the structure becomes long-range ordered. The LS transition brings upon a huge thermal response in addition to the incipient one. As a result, the colossal BC effect occurs in $n$-alkanes.

For the most solid-state $\mathrm{BC}$ materials (such as $\mathrm{AgI}, \mathrm{ANMn}_{3}, \mathrm{Ni}_{1-x} \mathrm{Fe}_{x} \mathrm{~S}$ and etc) ${ }^{12,15,16,20}$, the thermal response to the external pressure is solely due to the first-order phase transition. However, as shown in Fig. 1D and E for $\mathrm{C}_{n} \mathrm{H}_{2 n+2}$, the incipient contribution can be close to the half of the total $\left|\Delta \mathrm{T}_{\mathrm{d}}\right|$. For instance, at $342 \mathrm{~K}$ for $\mathrm{C}_{16} \mathrm{H}_{34}$, $\left|\Delta T_{\mathrm{d}}\right|$ is about $20 \mathrm{~K}$ at $293 \mathrm{MPa}$. At $367 \mathrm{MPa}$, the pressure-induced LS transition 
happens and $\left|\Delta T_{\mathrm{d}}\right|$ jumps to $47 \mathrm{~K}$. The incipient BC effect could be inherent to liquids that are much more compressible than solids, which favors a colossal BC effect based on LS transition.

The $T_{\mathrm{LS}}$ of $n$-alkanes $\mathrm{C}_{n} \mathrm{H}_{2 n+2}$ is strongly dependent on the $n$ value and can range from $\sim 247 \mathrm{~K}$ to $\sim 356 \mathrm{~K}$ when $n$ increases from 11 to 40 , which supplies a large temperature window for $\mathrm{BC}$ applications ${ }^{23,25,26}$. Besides, the $n$-alkanes have the advantages of self-nucleation to avoid supercooling, non-corrosiveness, long-term chemical stability without segregation, and commercial availability at reasonable $\operatorname{costs}^{22}$. Nowadays, $n$-alkanes, as the main component of paraffin, have been widely used for the storage of solar thermal energy in buildings, heat pumps, and spacecraft ${ }^{22-}$

24 . Practically, paraffin wax can be sealed into capsules with variable shapes to improve the heat transfer rate and avoid liquid leakage, and metal particles or flakes were often added in paraffin to improve its thermal conductivity ${ }^{22,23}$. As a caloric refrigerant, $n$ alkanes may also need to be sealed in elastic containers (plastic tubes in our measurement) so that external pressure can penetrate through. Therefore, many technical solutions for thermal energy storage based on paraffin can be readily invoked for the applications of $n$-alkanes as refrigerants of caloric cooling in future.

In summary, we reported the colossal BC effect near room temperature in $n$-alkanes $\mathrm{C}_{n} \mathrm{H}_{2 n+2}(n=14,16$ and 18$)$. The $n$-alkanes exhibit extremely huge thermal responses to pressure near room temperature, which outperforms those ever reported in any types of caloric materials. The theoretical calculations based on DFT and MD methods indicate a significant reduction of configuration entropy at low pressures, which causes an incipient $\mathrm{BC}$ effect. When the external pressure is strong enough to induce the LS transition, the long-range structure is formed and thus the configuration entropy is fully suppressed. Both mechanisms work together, leading to the colossal $\mathrm{BC}$ effect. The excellent BC performance, tunable operating temperatures, lost-cost raw materials, good environmental friendliness and well-known thermal properties suggest $n$-alkanes are promising refrigerants for caloric cooling. This work also indicates that more excellent BC materials could be obtained based on the LS transition materials in the near future.

\section{Methods}

DTA measurement. The Pressure-DTA equipment was constructed for calorimetry measurement under different hydrostatic pressures. One end of K-type thermocouple along with $\mathrm{C}_{n} \mathrm{H}_{2 n+2}$ was sealed in a separate plastic capsule, and the other end along with the reference compound (i.e. $\mathrm{C}_{n} \mathrm{H}_{2 n+2}$ with different $n$ ) was sealed in another capsule. Then they all were put into the DTA cell made of Teflon, using Daphne oil 7373 as the pressure medium. The Teflon cell was inserted in a $\mathrm{Be}-\mathrm{Cu}$ based pressure cylinder. The hydrostatic pressure was applied by the hydraulic machine. The annular heating sheet is adhered to the outer surface of the cylinder, which is used for the temperature control in liquid nitrogen dewar. During the experiment, the heating and cooling rate was set at $1 \mathrm{~K} / \mathrm{min}$. The entropy change at the first order phase transition under ambient pressure was evaluated by the differential scanning calorimeter (DSC). Based on the DSC and DTA results, the entropy change of first-order phase transition under different pressures can be calculated. 
Finally, the applied pressure will be corrected according to the measured phase transition temperature and the reported transition temperature-pressure phase diagram ${ }^{26,27}$.

Adiabatic temperature change measurement. When it comes to the direct measurement of the adiabatic temperature change, the same pressure apparatus used in DTA measurement was employed. Due to the high liquidity in liquid and extreme low modulus, the $\mathrm{C}_{n} \mathrm{H}_{2 n+2}$ itself can serve as the pressure medium. So, one end of K-type thermocouple along with $\mathrm{C}_{n} \mathrm{H}_{2 n+2}$ was sealed in the DTA cell made of Teflon, and the other end was embedded in the $\mathrm{Be}-\mathrm{Cu}$ based pressure cylinder. The measurements were carried out at the desired temperatures in cooling process and the data was collected in both pressure loading and unloading processes. Pressure was applied quickly to target values by manual means and maintained stably throughout the heat release cycle. Then the pressure relief value was opened and the pressure was released. The present hydraulic machine using the single-acting cylinder can realize the instant release of pressure, which is much faster than the pressure loading process.

Theoretical calculation methods. To investigate the vibrational properties of $\mathrm{C}_{18} \mathrm{H}_{38}$ in the solid phase, we calculate the force constants using the finite displacement method implemented in the Vienna Ab initio Simulation Package (VASP) ${ }^{44}$ and the PHONOPY code ${ }^{45}$. The $2 \times 2 \times 2$ supercell of fully-relaxed $\mathrm{C}_{18} \mathrm{H}_{38}$ structure containing 448 atoms is constructed. The Brillouin zone (BZ) was sampled with an $8 \times 8 \times 2 k$-point mesh for the structural relaxation of unit-cell and a $5 \times 5 \times 1 k$-point mesh for the supercell calculations. The exchange-correlation interaction was treated by generalized gradient approximation (GGA), which is parameterized by Perdew-Burke-Ernzerhof (PBE) ${ }^{47}$. We use the Grimme DFT-D3 method to describe the long-range van der Waals (VDW) interactions ${ }^{48}$. The cut-off energy of $500 \mathrm{eV}$ was used for the plane-wave basis expansion. The lattice constants and ion positions are optimized using the Broyden-Fletcher-Goldfarb-Shanno (BFGS) quasiNewton algorithm with the force convergence criterion less than $10^{-3} \mathrm{eV} / \AA$. The vibrational entropy is calculated using $S_{\text {vib }}=\frac{1}{2 T} \sum_{q v} \hbar \omega(\sigma / q v) \operatorname{coth}\left(\hbar w(\sigma / q v) / 2 k_{B} T\right)-k_{B} \sum_{q v} \ln [2 \sinh (\hbar w(\sigma /$ $\left.q v) / 2 k_{B} T\right)$ ], where $\omega$ is the vibrational frequency, $q$ the wave vector, and $v$ the index of the phonon mode ${ }^{46}$. Here we should point out that we calculated the phonon and vibrational entropy with ignoring the thermal expansion effect, since the calculations incorporating the thermal expansion effect would require the third-order force constants, which is quite time-consuming for the $\mathrm{C}_{18} \mathrm{H}_{38}$ supercell. To some extent, we may underestimate the vibrational entropy at high temperatures.

To get insight into the micro-process of solid-liquid phase change and the thermodynamic parameters, we perform the classic molecular dynamics (MD) simulations using the LAMMPS package with periodic boundary condition ${ }^{40}$. The adaptive intermolecular reactive empirical bond order (AIREBO) potential for a system of $\mathrm{C}-\mathrm{H}$ atoms was used in our MD simulations ${ }^{41}$. The Newton equation of motion was integrated by the velocity Verlet algorithm and the time-step is 0.5 fs. We used the coexisting solid-liquid phases to simulate the melting temperature of $\mathrm{C}_{18} \mathrm{H}_{38}$, since the two-phase method is more accurate for the melting simulation ${ }^{42,43}$. To construct the coexisting solid-liquid phases, we first performed the MD simulation started with the $7 \times 7 \times 3$ supercell $(8232$ atoms) in the $N P T$ ensemble at $200 \mathrm{~K}$ to obtain the solid phase (1,000,000 time steps), and then heated the solid phase to $550 \mathrm{~K}$ (far beyond the melting point) to obtain the liquid phase $(1,000,000$ time steps). The obtained solid and liquid phases were constructed into the coexisting phase include solid-liquid interface. We performed the MD simulation for the coexisting solid-liquid phases in the 
$N V E$ ensemble $(1,000,000$ time steps) to obtain the melting temperature. However, the two-phase method cannot obtain the useful thermodynamic data of $\mathrm{C}_{18} \mathrm{H}_{38}$ from the solid to liquid phase during heating. We have to use the normal one-phase method, although it usually overestimates the melting temperature due to the superheating problem. Here we performed the MD simulations by directly heating the solid phase of the $7 \times 7 \times 3$ supercell from $50 \mathrm{~K}$ to $550 \mathrm{~K}$ in the $N P T$ ensemble for $15 \mathrm{~ns}$ (30,000,000 time steps). We evaluated the entropy change $\Delta S$ from the enthalpy change $\Delta H$ of solid-liquid phases by using $\Delta S=\Delta H / T_{\mathrm{m}}$. Since the obtained temperature range of phase change is not narrow, the theoretically estimated entropy change may be inaccurate to a certain extent. However, it would not hinder us to analysis the law of the phase change under pressure comparing with the experimental data. The radial distribution function (RDF) and the bond-angle statistics are using RINGS code from the MD results ${ }^{49}$.

\section{Data availability}

All relevant data are presented via this publication and Supplementary Information.

\section{References}

1. P. Purohit, L. Höglund-Isaksson, J. Dulac, N. Shah, M. Wei, P. Rafaj, W. Schöpp, Electricity savings and greenhouse gas emission reductions from global phase-down of hydrofluorocarbons. Atoms. Chem. Phys. 20, 305-11327 (2020).

2. N. Abas, A. R. Kalair, N. Khan, A. Haider, Z. Saleem, M. S. Saleem, Natural and synthetic refrigerants, global warming: A review. Renew. Sust. Energ. Rev. 90, 57-569 (2018).

3. A. M. Fernando, P. F. Bernath, C. D. Boone, Trends in atmospheric HFC-23 $\left(\mathrm{CHF}_{3}\right)$ and HFC134a abundaces. J. Quant. Spectrosc. Ra. 238, 106540 (2019).

4. Y. B. Wang, S. C. Liu, V. Nian, X. Q. Li, J. Yuan, Life cycle cost-benefit analysis of refrigerant replacement based on experience from a supermarket project. Energy 187, 115918 (2019).

5. X. Moya, S. Kar-Narayan, N.D. Mathur, Caloric Materials near Ferroic Phase Transitions. Nat. Mater. 13, 439-450 (2014).

6. L. Manosa, A. Planes, Materials with Giant Mechanocaloric Effects: Cooling by Strength. Adv. Mater. 29, 1603607 (2017).

7. X. Moya, N. D. Mathur, Caloric materials for cooling and heating. Science 370, 797-803 (2020).

8. P. Lloveras, A. Aznar, M. Barrio, P. Negrier, C. Popescu, A. Planes, L. Manosa, E. Stern-Taulats, A. Avramenko, N.D. Mathur, X. Moya, J.L. Tamarit, Colossal Barocaloric Effects Near Room Temperature in Plastic Crystals of Neopentylglycol. Nat. Commun. 10, 1803 (2019).

9. B. Li, Y. Kawakita, S. Ohira-Kawamura, T. Sugahara, H. Wang, J.F. Wang, Y.N. Chen, S.I. Kawaguchi, S. Kawaguchi, K. Ohara, K. Li, D.H. Yu, R. Mole, T. Hattori, T. Kikuchi, S.I. Yano, Z. Zhang, Z. Zhang, W.J. Ren, S.C. Lin, O. Sakata, K. Nakajima, Z.D. Zhang, Colossal Barocaloric Effects in Plastic Crystals. Nature 567, 506-510 (2019).

10. A. Aznar, P. Lloveras, M. Barrio, P. Negrier, A. Planes, L. Mañosa, N. D. Mathur, X. Moya, J. -L. Tamarit, Reversible and irreversible colossal barocaloric effects in plastic crystals. J. Mater. Chem. A 8, 639-647 (2020).

11. F. B. Li, M. Li, X. Xu, Z. C. Yang, H. Xu, C. K. Jia, K. Li, J. He, B. Li, H. Wang, Understanding colossal barocaloric effect in plastic crystals. Nat. Commun. 11, 4190 (2020).

12. J. C. Lin, P. Tong, X. K. Zhang, Z. C. Wang, Z. Zhang, B. Li, G. H. Zhong, J. Chen, Y. D. Wu, H. L. Lu, L. H. He, B. Bai, L. S. Ling, W. H. Song, Z. D. Zhang, Y. P. Sun, Giant room- 
temperature barocaloric effect at the electronic phase transition in $\mathrm{Ni}_{1-x} \mathrm{Fe}_{x} \mathrm{~S}$. Mater. Horiz. 7, 2690-2695 (2020).

13. A. Aznar, P. Lloveras, J.-Y. Kim, E. Stern-Taulats, M. Barrio, J.L. Tamarit, C.F. Sanchez-Valdes, J.L. Sanchez Llamazares, N.D. Mathur, X. Moya, Giant and Reversible Inverse Barocaloric Effects near Room Temperature in Ferromagnetic $\mathrm{MnCoGeB}_{0.03}$. Adv. Mater. 31, 1903577 (2019).

14. L. Manosa, D. Gonzalez-Alonso, A. Planes, M. Barrio, J.L. Tamarit, I.S. Titov, M. Acet, A. Bhattacharyya, S. Majumdar, Inverse Barocaloric Effect in the Giant Magnetocaloric La-Fe-SiCo compound. Nat. Commun. 2, 595 (2011).

15. D. Matsunami, A. Fujita, K. Takenaka, M. Kano, Giant Barocaloric Effect Enhanced by the Frustration of the Antiferromagnetic Phase in $\mathrm{Mn}_{3} \mathrm{GaN}$. Nat. Mater. 14, $73-78$ (2015).

16. D. Boldrin, E. Mendive-Tapia, J. Zemen, J. B. Staunton, T. Hansen, A. Aznar, J.L. Tamarit, M. Barrio, P. Lloveras, J. Kim, X. Moya, L.F. Cohen, Multisite Exchange-Enhanced Barocaloric Response in $\mathrm{Mn}_{3}$ NiN. Phys. Rev. X 8, 041035 (2018).

17. J. M. Bermudez-Garcia, M. Sanchez-Andujar, S. Castro-Garcia, J. Lopez-Beceiro, R. Artiaga, M. A. Senaris-Rodriguez, Giant Barocaloric Effect in the Ferroic Organic-inorganic Hybrid $[\mathrm{TPrA}]\left[\mathrm{Mn}(\mathrm{dca})_{3}\right]$ Perovskite Under Easily Accessible Pressures. Nat. Commun. 8, 15715 (2017).

18. S.P. Vallone, A.N. Tantillo, A. M. Dos Santos, J.J. Molaison, R. Kulmaczewski, A. Chapoy, P. Ahmadi, M.A. Halcrow, K.G. Sandeman, Giant Barocaloric Effect at the Spin Crossover Transition of a Molecular Crystal. Adv. Mater. 31, 1807334 (2019).

19. L. Manosa, D. Gonzalez-Alonso, A. Planes, E. Bonnot, M. Barrio, J.L. Tamarit, S. Aksoy, M. Acet, Giant Solid-state Barocaloric Effect in the Ni-Mn-In Magnetic Shape-memory Alloy. Nat. Mater. 9, 478 (2010).

20. A. Aznar, P. Lloveras, M. Romanini, M. Barrio, J.L. Tamarit, C. Cazorla, D. Errandonea, N.D. Mathur, A. Planes, X. Moya, L. Manosa, Giant Barocaloric Effects Over a Wide Temperature Range in Superionic Conductor AgI. Nat. Commun. 8, 1851 (2017).

21. P. Lloveras, E. Stern-Taulats, M. Barrio, J.L. Tamarit, S. Crossley, W. Li, V. Pomjakushin, A. Planes, L. Manosa, N.D. Mathur, X. Moya, Giant Barocaloric Effects at Low Pressure in Ferrielectric Ammonium Sulphate. Nat. Commun. 6, 8801 (2015).

22. H. Jouhara, A. Żabnieńska-Góra, N. Khordehgah, D. Ahmad, T. Lipinski, Latent thermal energy storage technologies and application: A review. Int. J. Therm. Sci. 5-6, 100039 (2020).

23. L. F. Cabeza, A. Castell, C. Barreneche, A. de Gracia, A. I. Fernández, Materials used as PCM in thermal energy storage in buildings: A review. Renew. Sust. Energ. Rev. 15, 1675-1695 (2011).

24. A. Sharma, V. V. Tyagi, C. R. Chen, D. Buddhi, Review on thermal energy storage with phase change materials and applications. Renew. Sust. Energ. Rev. 13, 318-345 (2009).

25. J. F. Messerly, G. B. Guthrie, S. S. Todd, H. L. Finke, Low-temperature thermal data for pentane, n-heptadecane, and n-octadecane. Revised thermodynamic functions for the n-alkanes, C5-C18. J. Chem. Eng. Data 12, 338-346 (1967).

26. R. R. Nelson, W. Webb, J. A. Dixon, First-order phase transition of six normal paraffins at elevated pressures. J. Chem. Phys. 3, 1756-1764 (1960).

27. H. O. Baled, D. Xing, H. Katz, D. Tapriyal, I. K. Gamwo, Y. Soong, B. A. Bamgbade, Y. Wu, K. Liu, M. A. Mchugh, R. M. Enick, Viscosity n-Hexadecane, n-Octadecane and n-Eicosane at pressures up to $243 \mathrm{MPa}$ and temperature up to $534 \mathrm{~K}$. J. Chem. Thermodynamics 72, 108-116 
(2014).

28. A. N. Dunaeva, D. V. Antsyshkin, O. L. Kuskov, Phase diagram of $\mathrm{H}_{2} \mathrm{O}$ : Thermodynamic functions of the phase transitions of high-pressure ices. Solar Syst. Res. 44 (2010) 202-222.

29. J. N. Li, D. Dunstan, X. J. Lou, A. Planes, L. Mañosa, M. Barrio, J. -L. Tamarit, P. Lloveras, Reversible barocaloric effects over a large temperature span in fullerite $\mathrm{C}_{60}$. J. Mater. Chem. A 8, 20354-20362 (2020).

30. S. Yuce, M. Barrio, B. Emre, E. Stern-Taulats, A. Planes, J. -L. Tamarit, Y. Mudryk, K. A. Gschneidner, Jr., V. K. Pecharsky, L. Mañosa, Barocaloric effect in the magnetocaloric prototype $\mathrm{Gd}_{5} \mathrm{Si}_{2} \mathrm{Ge}_{2}$. App. Phys. Lett. 101, 071906 (2012).

31. T. Samanta, P. Lloveras, A. U. Saleheen, D. L. Lepkowski, E. Kramer, I. Dubenko, P. W. Adams, D. P. Young, M. Barrio, J. -L. Tamarit, N. Ali, S. Stadler, Barocaloric and magnetocaloric effects in $(\mathrm{MnNiSi})_{1-x}(\mathrm{FeCoGe})_{x}$. App. Phys. Lett. 112, 021907 (2018).

32. R. R. Wu, L. F. Bao, F. X. Hu, H. Wu, Q. Z. Huang, J. Wang, X. L. Dong, G. N. Li, J. R. Sun, F. R. Shen, T. Y. Zhao, X. Q. Zheng, L. C. Wang, Y. Liu, W. L. Zuo, Y. Y. Zhao, M. Zhang, X. C. Wang, C. Q. Jin, G. H. Rao, X. F. Han, B. G. Shen, Giant barocaloric effect in hexagonal $\mathrm{Ni}_{2}$ In-type Mn-CoGe-In compounds around room temperature. Sci. Rep. 5, 18027 (2015).

33. E. Stern-Taulats, A. Planes, P. Lloveras, M. Barrio, J. -L. Tamarit, S. Pramanick, S. Majumdar, C. Frontera, L. Mañosa, Barocaloric and magnetocaloric effects in $\mathrm{Fe}_{49} \mathrm{Rh}_{51}$. Phys. Rev. B 89, 214105 (2014).

34. E. Stern-Taulats, P. Lloveras, M. Barrio, E. Defay, M. Egilmez, A. Planes, L. Mañosa, N. D. Mathur, X. Moya, Inverse barocaloric effects in ferroelectric $\mathrm{BaTiO}_{3}$ ceramics. APL Mater. 4, 091102 (2016).

35. A. Aznar, A. Gràcia-Condal, A. Planes, P. Lloveras, M. Barrio, J. -L. Tamarit, W. X. Xiong, D. Y. Cong, C. Popescu, L. Mañosa, Giant barocaloric effect in all-d-metal Heusler shape memory alloys. Phys. Rev. Mater. 3, 044406 (2019).

36. Z. Y. Wei, Y. Shen, Z. Zhang, J. P. Guo, B. Li, E. K. Liu, Z. D. Zhang, J. Liu, Low-pressureinduced giant barocaloric effect in an all-d-metal Husler $\mathrm{Ni}_{35.5} \mathrm{Co}_{14.5} \mathrm{Mn}_{35} \mathrm{Ti}_{15}$ magnetic shape memory alloy. APL Mater. 8, 051101 (2020).

37. W. Imamura, E. O. Usuda, L. S. Paixão, N. M. Bom, A. M. Gomes, A. M. G. Carvalho, Supergiant barocaloric effect in acetoxy silicone rubber over a wide temperature range: great potential for solid-state cooling. Chinese J. Polym. Sci. 38, 999-1005 (2020).

38. N. M. Bom, W. Imamura, E. O. Usuda, L. S. Paixão, A. M. G. Carvalho, Giant barocaloric effect in natural rubber: a relevant step toward solid-state cooling. ACS Macro Lett. 7, 31-36 (2018).

39. C. M. Miliante, A. M. Christmann, E. O. Usuda, W. Imamura, L. S. Paixão, A. M. G. Carvalho, A. R. Muniz, Unveiling the origin of the giant barocaloric effect in natural rubber. Macromolecules 53, 2606-2615 (2020).

40. S. Plimpton, Fast parallel algorithms for short-range molecular dynamics. J. Comput. Phys. 117, 1-19 (1995).

41. S. J. Stuart, A. B. Tutein, and J. A. Harrison, A reactive potential for hydrocarbons with intermolecular interactions. J. Chem. Phys. 112, 6472-6486 (2000).

42. S. Yoo, X. C. Zeng, J. R. Morris, The melting lines of model silicon calculated from coexisting solid-liquid phases. J. Chem. Phys. 120, 1654-1656 (2004).

43. A. Marbeuf, R. Brown, Molecular dynamics in n-alkanes: Premelting phenomenon and rotator phases. J. Chem. Phys. 124, 054901 (2006).

44. G. Kresse and D. Joubert, From ultrasoft pseudopotentials to the projector augmented-wave method. Phys. Rev. B 59, 1758-1775 (1999).

45. A. Togo and I. Tanaka, First principles phonon calculations in materials science. Scr. Mater. 108, 
$1-5(2015)$.

46. Y. L. Wang, Y. S. Zhang, and C. Wolverton, First-principles studies of phase stability and crystal structures in Li-Zn mixed-metal borohydrides. Phys. Rev. B 88, 024119 (2013).

47. J. P. Perdew, K. Burke, M. Ernzerhof, Generalized gradient approximation made simple. Phys. Rev. Lett. 77, 3865-3868 (1996).

48. S. Grimme, J. Antony, S. Ehrlich, H. Krieg, A consistent and accurate ab initio parametrization of density functional dispersion correction (DFT-D) for the 94 elements H-Pu. J. Chem. Phys. 132, 154104 (2010).

49. S. Le Roux, P. Jund, Ring statistics analysis of topological networks: New approach and application to amorphous $\mathrm{GeS}_{2}$ and $\mathrm{SiO}_{2}$ systems. Comp. Mat. Sci. 49, 70-83 (2010).

\section{Additional information}

Supplementary information accompanies this paper at https://doi.org/.....

Competing interests: The authors declare no competing interests.

Reprints and permissions information is available online at www.nature.com/reprints.

Journal peer review information: Nature Communications thanks the anonymous reviewer for their contribution to the peer review of this work. Peer reviewer reports are available.

Publisher's note: Springer Nature remains neutral with regard to jurisdictional claims in published maps and institutional affiliations. 
Fig. 1. The adiabatic temperature change measured by direct method $\left(\Delta T_{d}\right)$ and the reversible isothermal entropy change $\left(\Delta S_{\mathrm{r}}\right)$. The sample's temperature change under different pressures at $294 \mathrm{~K}(\mathrm{~A}), 305 \mathrm{~K}(\mathrm{~B})$ and $356 \mathrm{~K}(\mathrm{C})$; All reliable $\Delta T_{\mathrm{d}}$ values for $\mathrm{C}_{18} \mathrm{H}_{38}(\mathrm{D})$ and $\mathrm{C}_{16} \mathrm{H}_{34}(\mathrm{E}) ; \Delta \mathrm{S}_{\mathrm{r}}$ under different pressures for $\mathrm{C}_{18} \mathrm{H}_{38}(\mathrm{~F})$ and $\mathrm{C}_{16} \mathrm{H}_{34}$ (G).
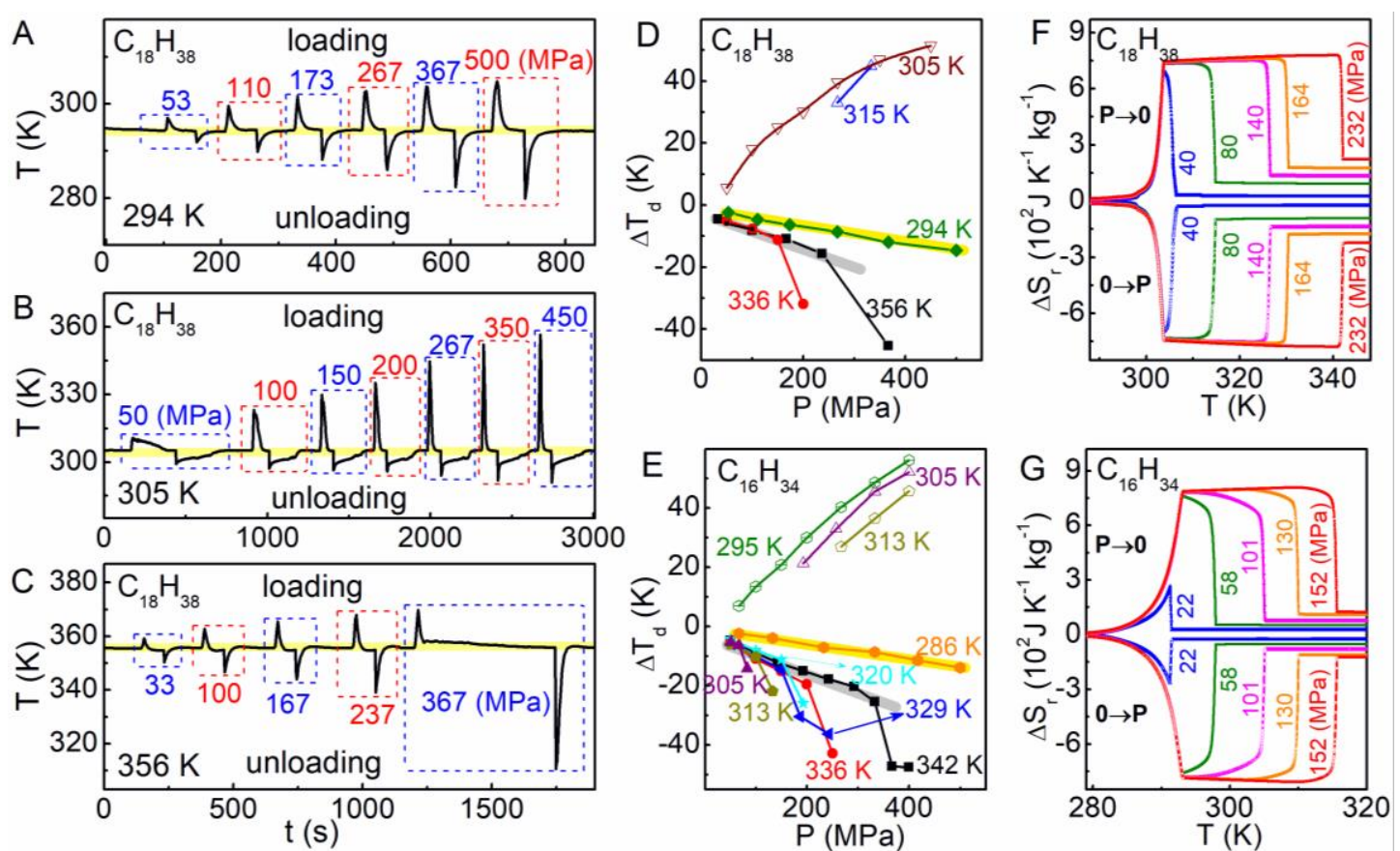
Fig. 2. The comparision of $\mathrm{BC}$ performance between $\mathrm{C}_{n} \mathrm{H}_{2 n+2}$ and existing $\mathrm{BC}$ materials. Maximum adiabatic temperature change (A) and isothermal entropy change (B) as a function of pressure for $\mathrm{C}_{18} \mathrm{H}_{38}, \mathrm{C}_{16} \mathrm{H}_{34}$ and $\mathrm{C}_{14} \mathrm{H}_{30}$, are shown along with the reported $\mathrm{BC}$ materials. Here, the isothermal entropy change in references is mainly obtained by indirect or quasi-direct methods. According to different methods, reversible $\left(\left|\Delta S_{\mathrm{r}}\right|\right)$ and non-reversible entropy changes $\left(\left|\Delta S_{\mathrm{ir}}\right|\right)$ can be obtained in literatures. For the adiabatic temperature changes, some were measured by direct measurement $\left(\left|\Delta T_{\mathrm{d}}\right|\right)$. Others were estimated by quasi-direct method, corresponding to either reversible $\left(\left|\Delta T_{\mathrm{r}}\right|\right)$ or irreversible values $\left(\left|\Delta T_{\mathrm{ir}}\right|\right)$.
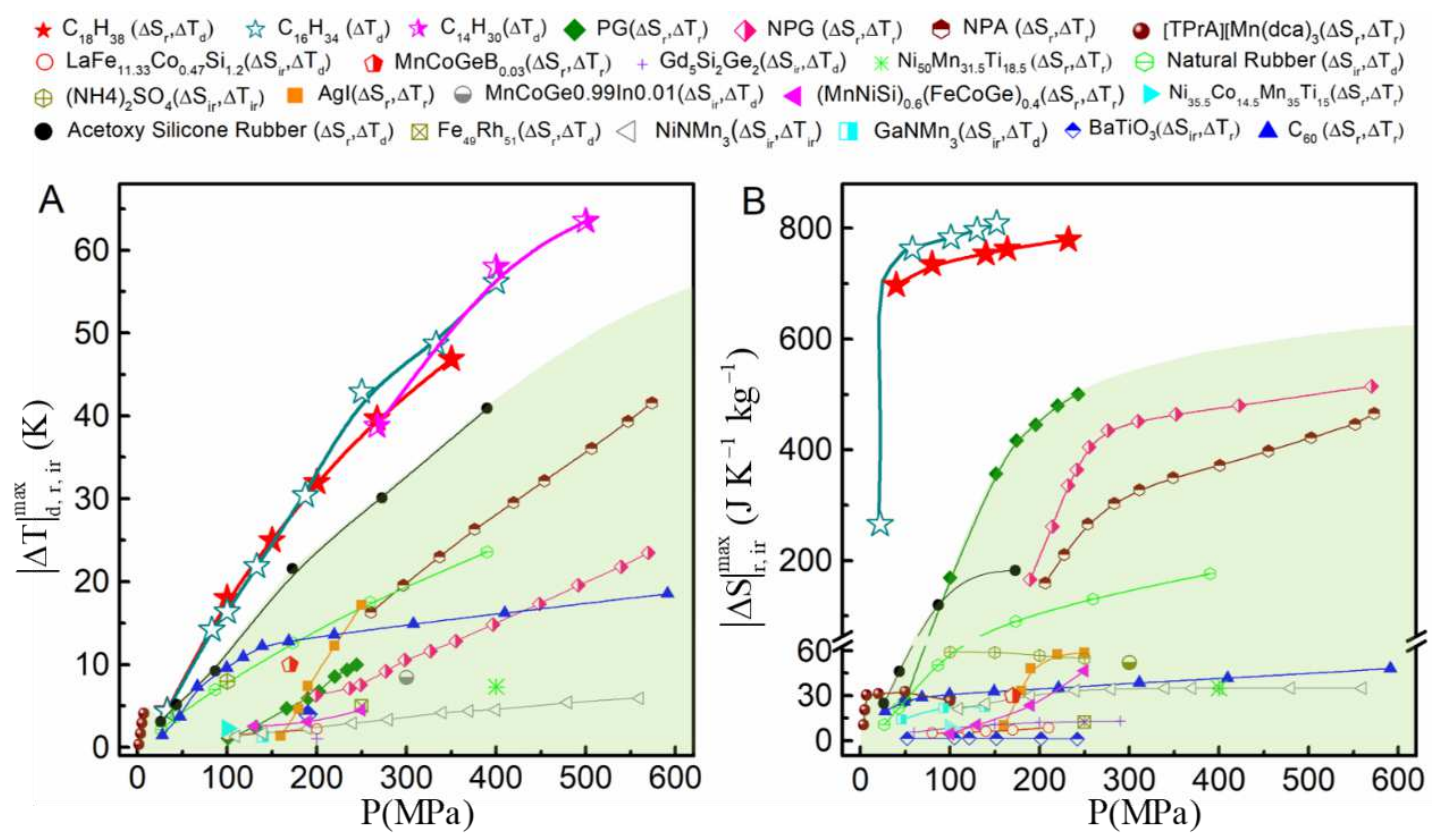
Fig. 3. The theoretical calculation of thermodynamical information under different pressures for $\mathbf{C}_{\mathbf{1 8}} \mathbf{H}_{\mathbf{3 8}}$. (A) The LS transition temperature ( $T_{\mathrm{LS}}$ ) and entropy change $\left(\Delta S_{\mathrm{LS}}\right)$ due to the LS transition under different pressures determined from both the experiment and theoretical calculation. (B) The temperature dependent lattice vibration entropy $\left(\Delta S_{\mathrm{vib}}\right)$ calculated by the DFT and the total entropy based on the experiment results. The structure of $\mathrm{C}_{18} \mathrm{H}_{38}$ under $0 \mathrm{MPa}$ and $200 \mathrm{MPa}$ at several typical temperatures including (C) $T_{M D}^{S}=300 \mathrm{~K}$, (D) $T_{M D}^{S}=400 \mathrm{~K}$, and (E) $T_{M D}^{S}=500 \mathrm{~K}$. The radial distribution function $(\mathrm{RDF})$ of $\mathrm{C}_{18} \mathrm{H}_{38}$ under different pressures at $(\mathrm{F}) T_{M D}^{S}=$ $300 \mathrm{~K},(\mathrm{G}) T_{M D}^{S}=400 \mathrm{~K}$, and $(\mathrm{H}) T_{M D}^{S}=500 \mathrm{~K}$.
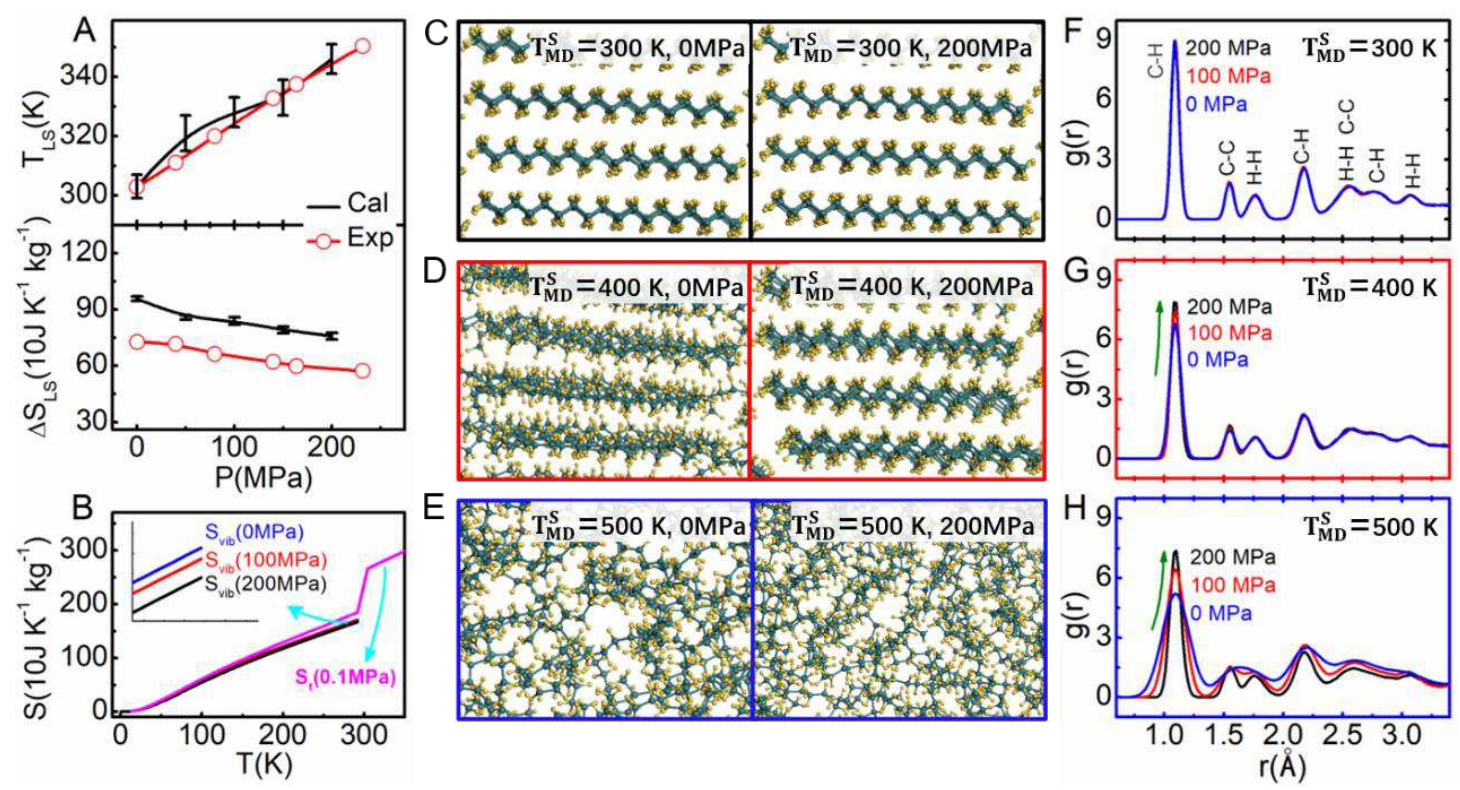
Figures
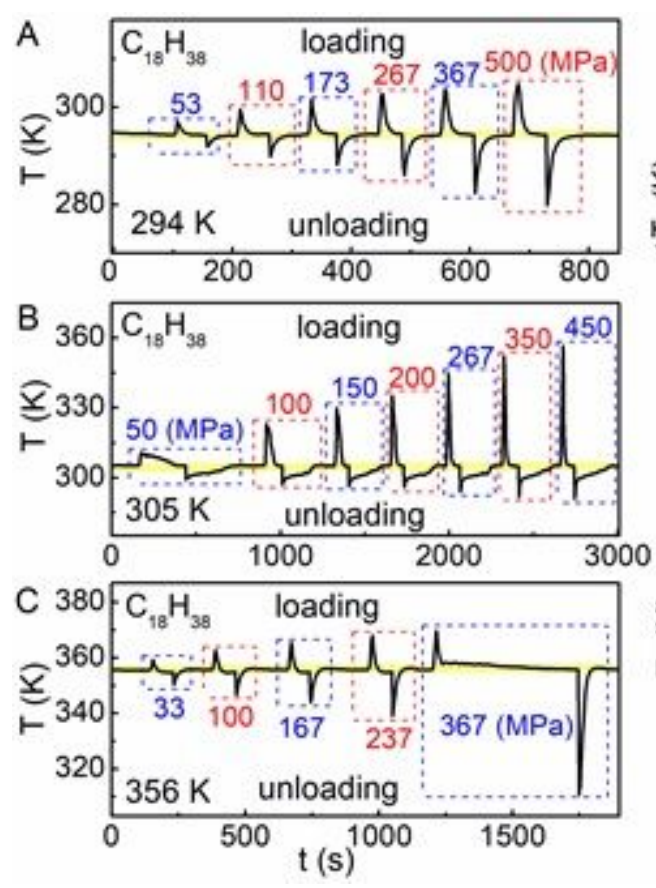
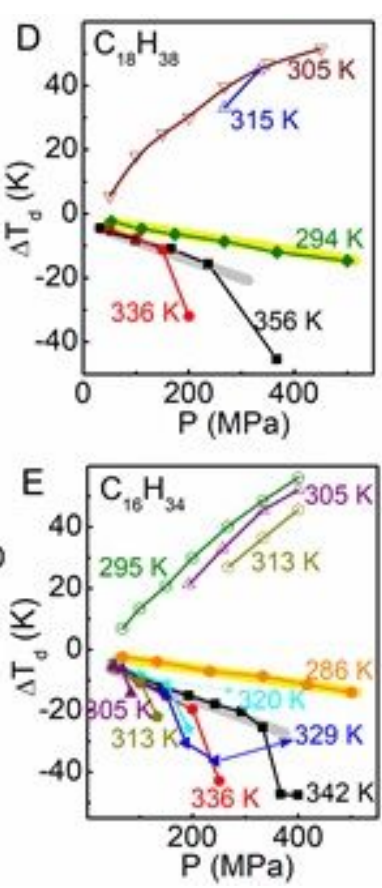
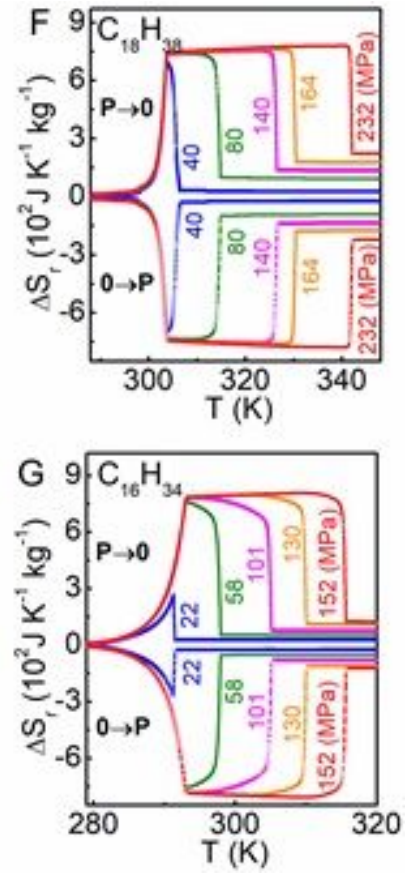

\section{Figure 1}

The adiabatic temperature change measured by direct method $(\Delta \mathrm{Td})$ and the reversible isothermal entropy change $(\Delta \mathrm{Sr})$. The sample's temperature change under different pressures at $294 \mathrm{~K}(\mathrm{~A}), 305 \mathrm{~K}(\mathrm{~B})$ and $356 \mathrm{~K}(\mathrm{C})$; All reliable $\Delta \mathrm{Td}$ values for $\mathrm{C} 18 \mathrm{H} 38$ (D) and $\mathrm{C} 16 \mathrm{H} 34(\mathrm{E}) ; \Delta \mathrm{Sr}$ under different pressures for $\mathrm{C} 18 \mathrm{H} 38(\mathrm{~F})$ and $\mathrm{C} 16 \mathrm{H} 34(\mathrm{G})$.

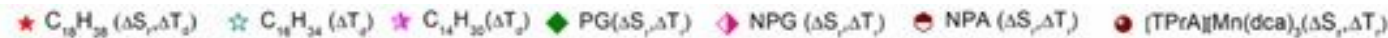

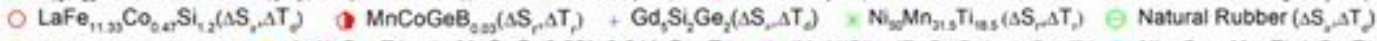

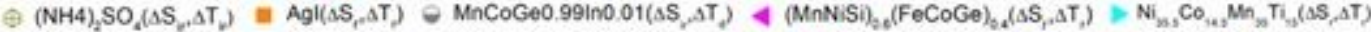

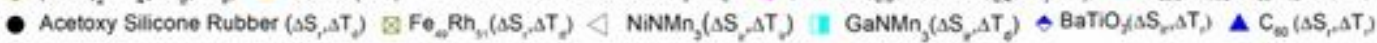
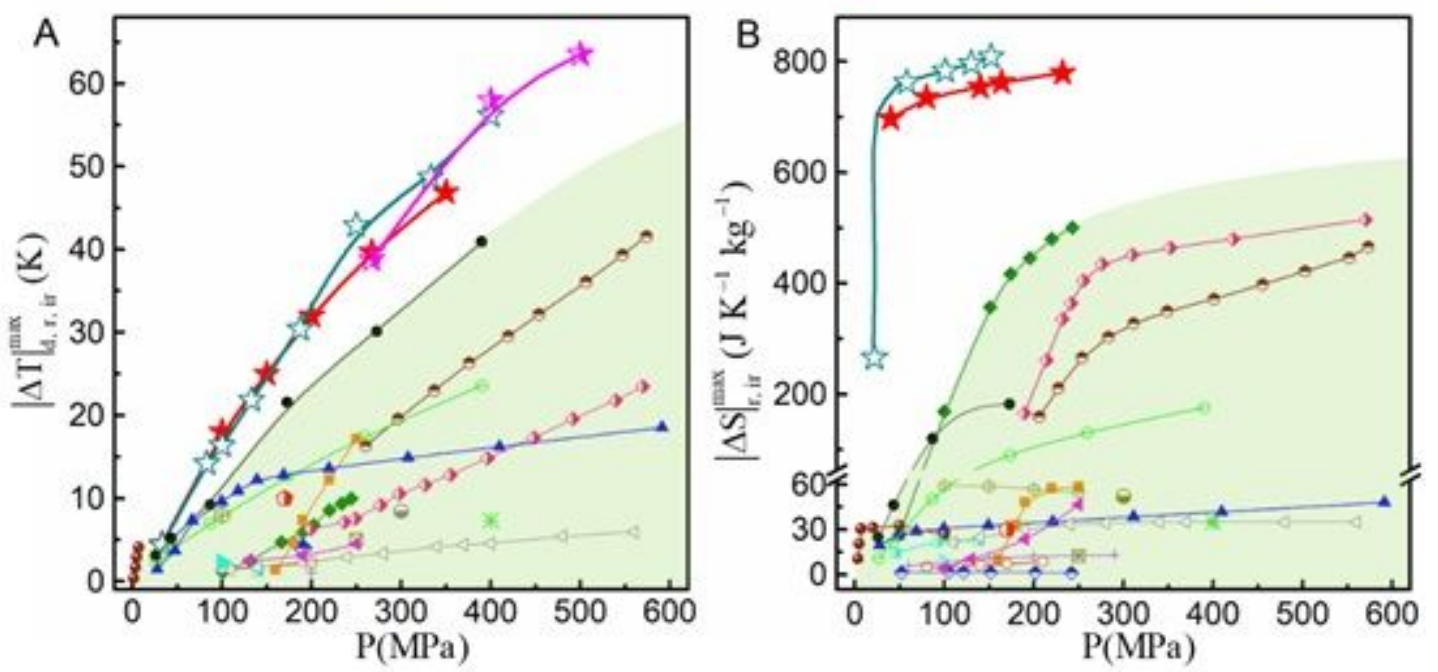

Figure 2 
The comparision of $\mathrm{BC}$ performance between $\mathrm{CnH} 2 \mathrm{n}+2$ and existing $\mathrm{BC}$ materials. Maximum adiabatic temperature change $(A)$ and isothermal entropy change $(B)$ as a function of pressure for $\mathrm{C} 18 \mathrm{H} 38$, $\mathrm{C} 16 \mathrm{H} 34$ and $\mathrm{C} 14 \mathrm{H} 30$, are shown along with the reported $\mathrm{BC}$ materials. Here, the isothermal entropy change in references is mainly obtained by indirect or quasi-direct methods. According to different methods, reversible $(|\Delta \mathrm{Sr}|)$ and non-reversible entropy changes $(|\Delta \mathrm{Sir}|)$ can be obtained in literatures. For the adiabatic temperature changes, some were measured by direct measurement $(|\Delta T d|)$. Others were estimated by quasi-direct method, corresponding to either reversible $(|\Delta \mathrm{Tr}|)$ or irreversible values $(|\Delta \mathrm{Tir}|)$.
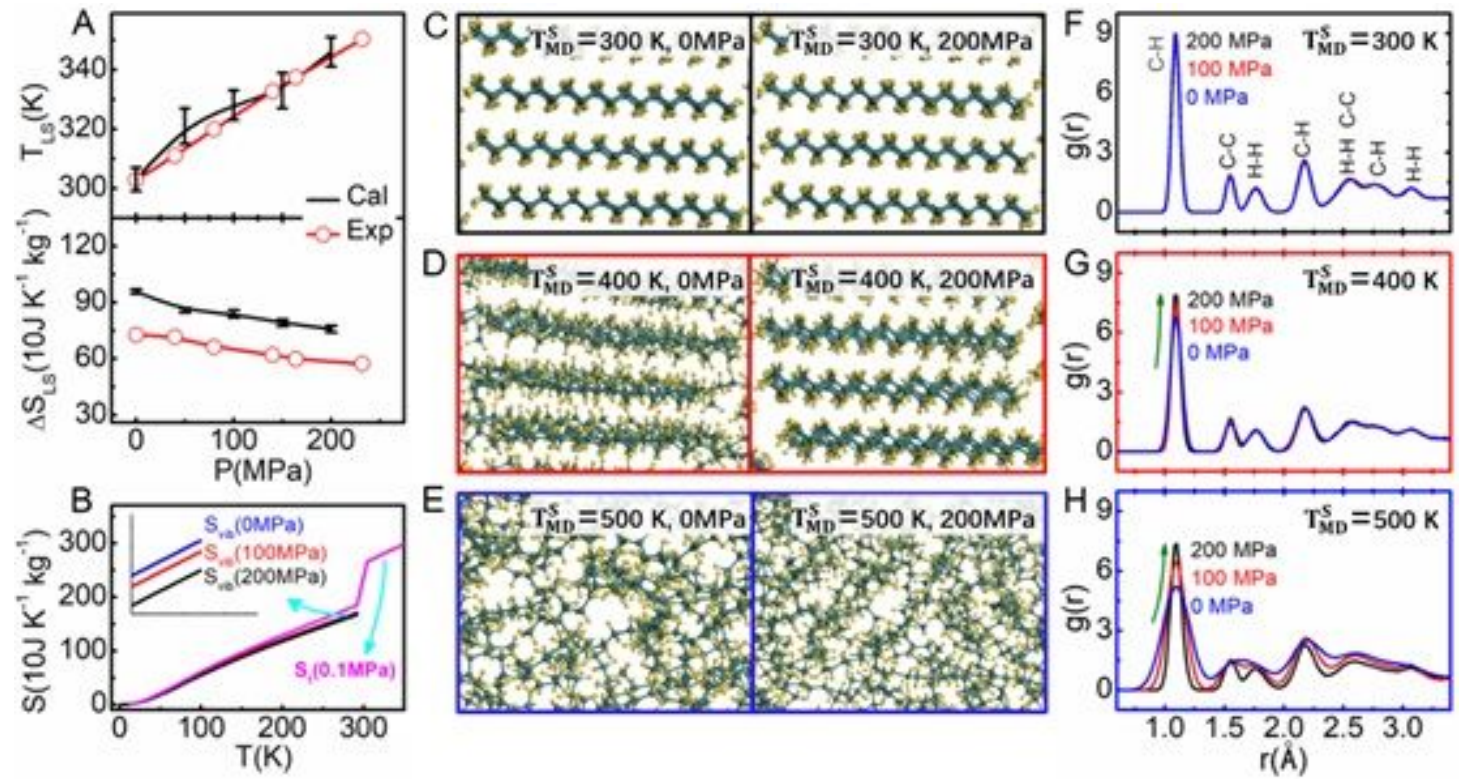

Figure 3

The theoretical calculation of thermodynamical information under different pressures for $\mathrm{C} 18 \mathrm{H} 38$. (A) The LS transition temperature (TLS) and entropy change ( $\Delta S L S)$ due to the LS transition under different pressures determined from both the experiment and theoretical calculation. (B) The temperature dependent lattice vibration entropy $(\triangle \mathrm{Svib})$ calculated by the DFT and the total entropy based on the experiment results. The structure of $\mathrm{C} 18 \mathrm{H} 38$ under $0 \mathrm{MPa}$ and $200 \mathrm{MPa}$ at several typical temperatures including $(C)=300 \mathrm{~K},(\mathrm{D})=400 \mathrm{~K}$, and $(\mathrm{E})=500 \mathrm{~K}$. The radial distribution function $(\mathrm{RDF})$ of $\mathrm{C} 18 \mathrm{H} 38$ under different pressures at $(F)=300 \mathrm{~K},(\mathrm{G})=400 \mathrm{~K}$, and $(\mathrm{H})=500 \mathrm{~K}$.

\section{Supplementary Files}

This is a list of supplementary files associated with this preprint. Click to download.

- SupplementaryVideo1.mp4

- SupplementaryInformation.docx 